\title{
Real Time Handwritten Marathi Numerals Recognition Using Neural Network
}

\author{
Sandeep B. Patil \\ Shri Shankaracharya Technical Campus, Bhilai, India \\ Email: patilsandeepb1212@gmail.com \\ G.R. Sinha, Dr. \\ Shri Shankaracharya Technical Campus, Bhilai, India \\ Emaildrgrsinha@ieee.org
}

\begin{abstract}
Character recognition is an important task in biometrics. This paper uses neural network for real time handwritten Marathi numerals recognition. We have taken 150 online Marathi numerals written in different styles by 10 different persons. Out of these, 50 numerals were used for training purpose and another 100 numerals were used for recognition purpose. The numerals undergo the preprocessing steps using image processing techniques and after character digitization it is further subjected to the multilayer backward propagation neural network for recognition purpose. The proposed research work gives recognition accuracy from $97 \%$ and to $100 \%$ for the different resolution of input vector.
\end{abstract}

Index Terms - Marathi, Recognition, Neural Network, Back Propagation, Epochs

\section{Introduction}

Handwriting processing is a domain under great scope of expansion and hence the interest devoted in this field is not explained only by the exciting challenges involved, but also the huge benefits that a system, designed in the context of a commercial application. Two classes of recognition systems are usually distinguished: online systems for which handwriting data are captured during the writing process, which makes available the information on the ordering of the strokes, and offline systems for which recognition takes place on a static image captured once the writing process is over. Online handwriting recognition allows for such input modalities. Online handwritten scripts are usually dealt with pen tip traces from pen-down to pen-up positions ${ }^{[1][2][3]}$. Neural Network and Hidden Markov Models are the popular, amongst the techniques which have been investigated for handwriting recognition. It has been observed that Neural Network in general obtained best results than Hidden Morkov models, when a similar feature set is applied. Here we have used Multi-Layer Perceptron
(MLP) neural network and trained with backpropagation which is the most popular and versatile forms of neural network classifiers and is also among the most frequently used traditional classifiers for handwriting recognition ${ }^{[3][4][5][6]}$.

\subsection{System Overview}

We propose to implement a simple optical Marathi numeral recognition system from (0-9). The system will take as input a scanned bitmap consisting of multiple isolated numerals.

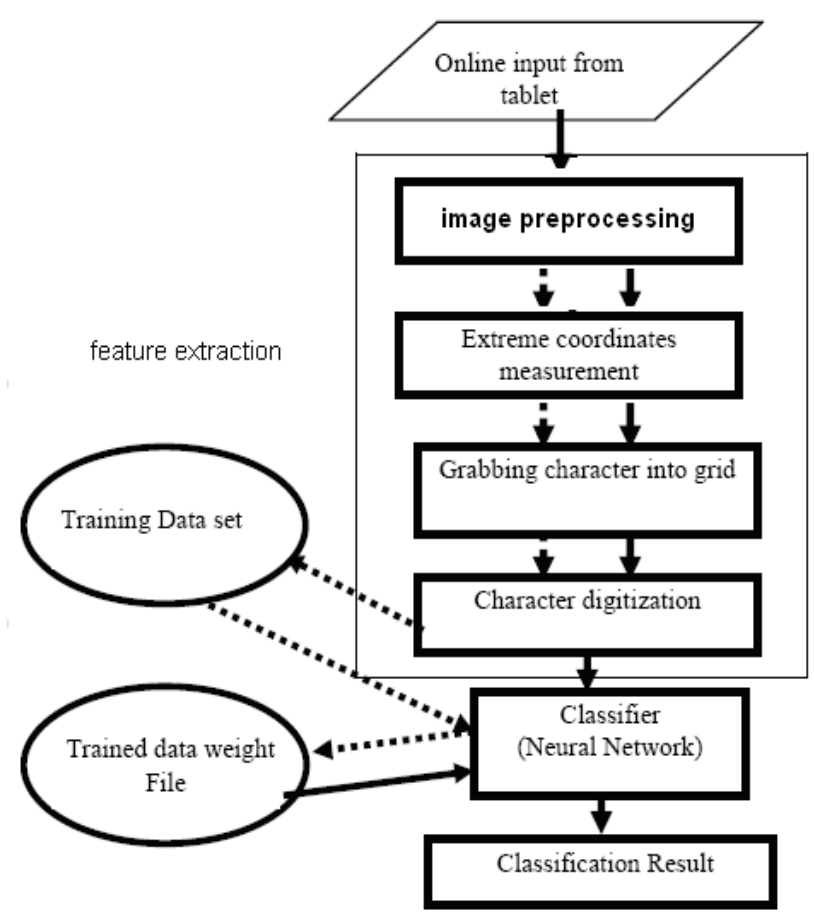

Fig. 1: Block diagram of the system

The system will then segment the image, and perform feature extraction and pattern classification on the individual objects. The resulting numeral will be output. A block diagram of the proposed online recognition systemof isolated Marathi digit is shown in Fig 1. 
The flow of data during training is shown by the dashed line arrows, while the data flow during recognition is shown by solid line arrows. The input to the system is a sequence of handwritten numerals patterns which is preprocessed. After preprocessing the input from tablet the extreme coordinates i.e. left, right, top, and bottom are calculated. Then numeral is captured in a grid and sensing the character pixels in grid boxes, the numeral is digitized in a binary string. This binary string is applied at the input of Neural Network for training and recognition. Grid size of $7 \times 5$ (i.e. 7 rows and 5 columns) were used in the experiments.

\section{Marathi Script, data acquisition and preprocessing}

Marathi is a form of alphabet called an abugida, as each consonant has an inherent vowel (A) that can be changed with different vowel signs. Vowels can be written as independent characters, or by using a variety of diacritical marks which are written above, below, before or after the consonants they belong to. Tablet has been used to take the samples from different subjects. Each subject was asked to write on tablet board (writing area). The acquired image is first being converted to grayscale image followed by the threshing technique, which makes the image binary image. The binary image is then goes through the connectivity test in order to check for the maximum connected component, which is in the form of box. After locating the box, the individual characters are then cropped into different sub images that are the raw data for the feature extraction routine. The following preprocessing steps are applied on the acquired image.

\subsection{Image Preprocessing}

The first step of this processing is to converts the acquired RGB image into gray image and then binary. The binary image is then inverted because when we are applying morphological operations such as edge detection which is applicable to black background i.e. with pixel ' 0 ' and white foreground with pixel ' 1 '. The main aim is to do that for simpler and faster calcu lations for further processing.

\subsection{Edge Detection:}

In practice, edge detection is performed in the spatial domain, because it is computationally less expensive and often yields better results. Since edges correspond to strong illumination gradients, we can high light them by calculating the derivatives of the image. In this numeral recognition process we are using the method of gradient edge detection with Sobel operator ${ }^{[6][7][8][10]}$. Gradient edge detection is the more widely used technique. Here, the image is convolved with only two masks, one estimating the gradient in the $\mathrm{x}$-d irection $\mathrm{Gx}$, the other the gradient in the y-direction Gy. The two masks are shown in "Fig. (2)" below. These masks are designed to respond maximally to edges running vertically and horizontally relative to the pixel grid, one mask for each of the two perpendicular orientations. The mask can be applied separately to the input image, to produce separate measurements of the gradient components in each orientation. These are then can be

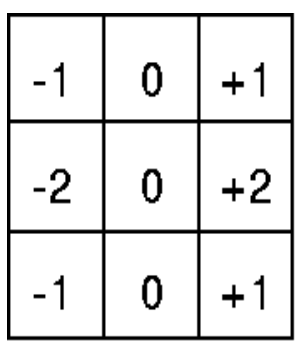

$G \times$

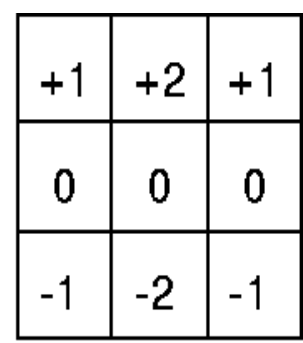

Gy
Fig. 2: Sobel convolution mask

Combined together to find the absolute magnitude of the gradient at each point and the orientation of that gradient. The gradient magnitude is given by

$$
\|G\|=\sqrt{G x^{2}+G y^{2}}
$$

The angle of orientation of the edge gives rise to the spatial gradient is given by

$$
\emptyset=\arctan \left(G_{y} \mid G x\right)-3 \pi / 4
$$

In this case, orientation ' 0 ' is taken to mean that the direction of maximum contrast from black to white runs from left to right on the image, and other angles are measured anti-clockwise from this. This absolute magnitude is the only output the user sees. The two components of the gradient are conveniently computed and added in a single pass over the input image using the pseudo-convolution operator as shown in "Fig. (3)".

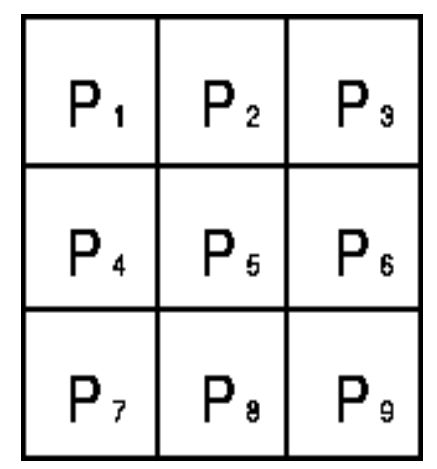

Fig.3: Pseudo-convolution masks used to compute approximate gradient magnitude

$$
\begin{aligned}
& \text { Using this mask the approximate magnitude is given } \\
& \text { by } \\
& \begin{array}{l}
|G|=(P 1+2 * P 2+P 3)-(P 7+2 * P 8+P 9) \|+ \\
\|(P 3+2 * P 6+P 9)-(P 1+2 * P 4+P 7)\|
\end{array}
\end{aligned}
$$
by 


\subsection{Dilation}

Dilation is typically applied over hear to gradually enlarge the boundaries of regions of the foreground pixels thus the area of foreground pixels grow in size while holes within this regions becomes smaller. The dilation operator takes two pieces of data as input. The first is the image which is to be dilated and the second is a set of coordinate point known as structuring element. The structuring element determines the precise effect of the dilation on the input image.

Mathematically, dilation of A by B is denoted by $\mathrm{A} \oplus \mathrm{B}$ and is defined as ${ }^{[7-10]}$.

$$
\mathrm{A} \oplus \mathrm{B}=\{Z \mid(B) z \cap A \neq \varnothing\}
$$

\subsection{Hole filling}

Hole filling is determined by selection of marker and mask images. Here, we choose the marker image is $f_{\mathrm{m}}$ to be ' 0 ' everywhere except on the image border, where it is set to ' 1 ' $[6,10-11]$.

$$
\left\{\begin{array}{cc}
1-f\left(x_{x} y\right) & \text { if } \left.\left(x_{x} y\right)\right) \text { is on the border of " } f^{\prime \prime} \\
0 & \text { atherwise }
\end{array}\right.
$$

Figure 4 show the above preprocessing steps followed on online data.

The sizes of the sub images are not fixed since they are exposed to noises which will affect the cropping process to be varied from one to another. This will cause the input of the network to become non-standard and hence, prohibit the data from feeding to the network. To solve this problem, the sub images have been resized to $50 \times 70$ and then finding the average value in each $10 \times 10$ block, the image can be down to $5 \times 7$ matrices, with fuzzy value and become 35 inputs for the network. Figure 5. Shows the object location.

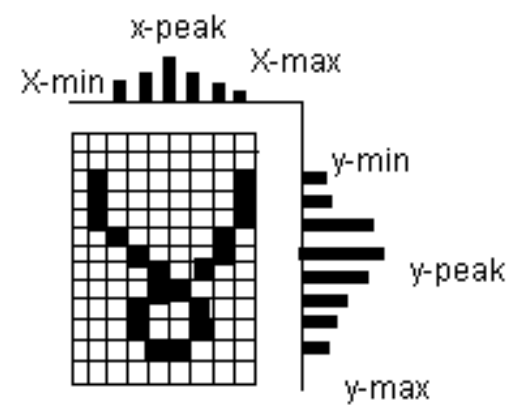

Fig. 6: Image standardization
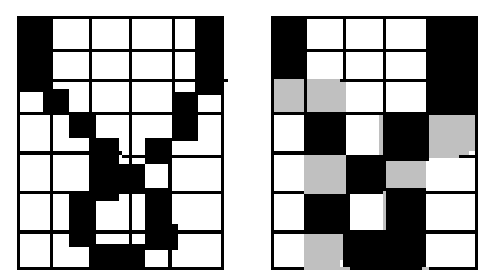

Fig. 7: Image resizing

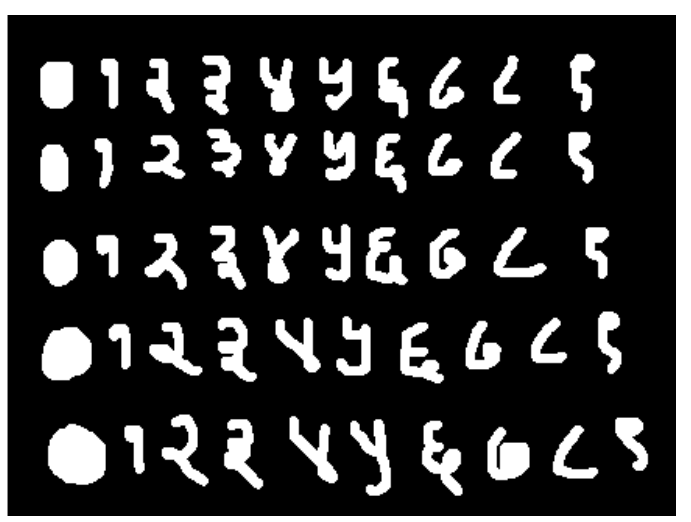

Fig. 4: Image dilation and hole filling

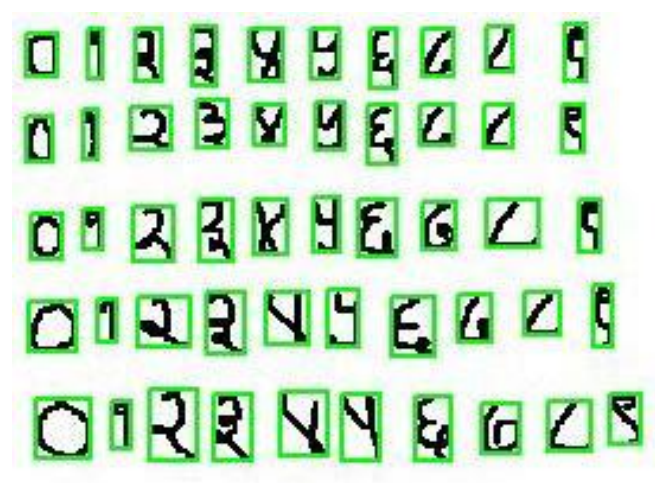

Fig. 5: Object location

\section{Feature Extraction and Character Detection}

The sub-images have to be cropped sharp to the border of the character in order to standardize the subimages. The image standardization is done by finding the maximum row and column with $1 \mathrm{~s}$ and with the peak point, increase and decrease the counter until meeting the white space, or the line with all $0 \mathrm{~s}$. This technique is shown in Fig. 6 with a numeral " $\varnothing$ " is being cropped and resize.

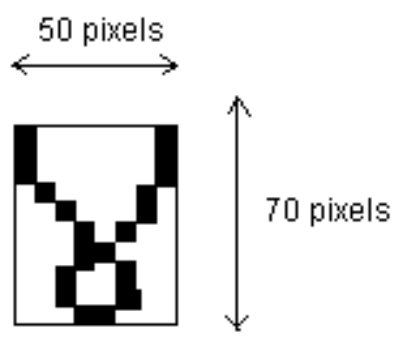

The image pre-processing is then followed by the image resize again to meet the network input require ment, 5 by 7 matrices, where the value of 1 will be assign to all pixels where all 10 by 10 box are filled with $1 \mathrm{~s}$, as shown in figure 7 . Finally the 5 by 7 matrices are concatenated into a stream so that it can be fed to the network 35 input neurons. The input of the network is actually a negative image of the figure, 
where the input range is 0 to 1 , with 0 equal to black and 1 indicate white, while the value in between shows the intensity of relevant pixels.

\section{Neural network and Training}

The network receives the 35 Boolean values as a 35 element vector. It is then required to identify the character by responding with a 10- element output vector. The Fig. 8 shows the back-propagation method for an artificial neural network with one hidden layer. Here 'o' will always denote a cell in the input layer, ' $p$ ' a cell in the hidden layer and ' $q$ ' a cell in the output layer.

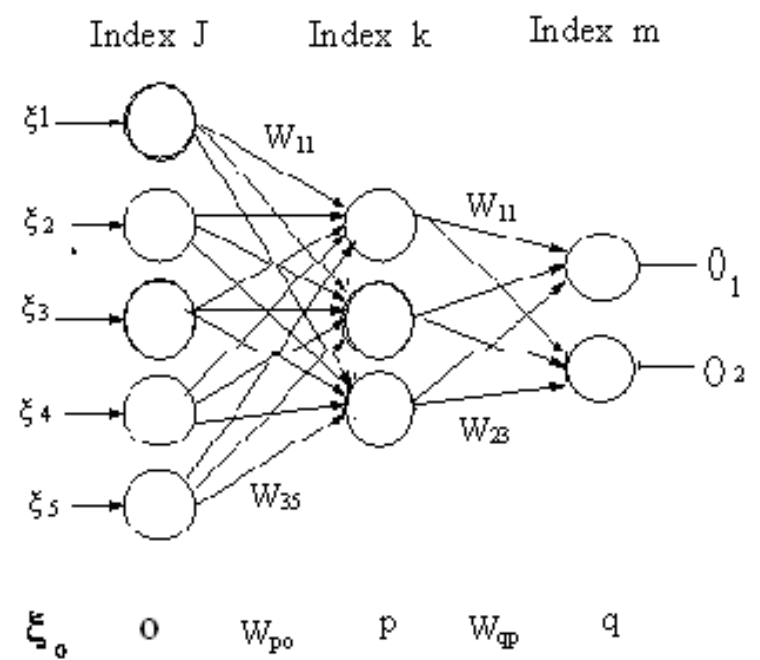

Fig. 8: Neural network with one hidden layer

Here $\xi_{0}$ denote the input at cell ' $o$ ', and $\mathrm{N}$ the number of input cells (i.e., $(\xi 1 \ldots \xi \mathrm{N})$ is the input vector), let ' $W s r$ ' be the weight of the link between cell ' $s$ ' in layer ' $l$ ' and cell ' $r$ ' in layer ' $l-1$ ', ' $I p$ ' the input and ' $O p$ ' the output of a cell ' $p$ ' in the hidden layer, and let ' $I q$ ' be the input and ' $O q$ ' be the output of a cell ' $q$ ' in the output layer. Furthermore, let ' $\mu$ ' denote an input pattern $\mu=1 \ldots . p$ index, (with $p$ being the total number of input patterns) and let the set $\left[\xi^{\mu}{ }_{0} \zeta^{\mu}\right]$ be the set of input-output patterns (with $\mu$ denoting the number of the pattern). Finally, let $j, k$ and $m$ be indices denoting cells in the input, hidden and output layer respectively [4-6].

\subsection{Neural Network in numerals recognition}

Neural network classifier exhibit powerful properties and they have been used in handwriting recognition particularly with digits, isolated characters, and words in small vocabularies. In this work, multilayer feedforward back propagation neural network has been implemented. The neural network had three layers: an input layer consisting of 35 nodes, a hidden layer consisting of 20 nodes, and an output layer 10 nodes one for each numerals ${ }^{[11-16]}$. Fig. 9 shows the three layer artificial neural network for numeral recognition.
The network uses back-propagation in addition to bias weight and momentum.

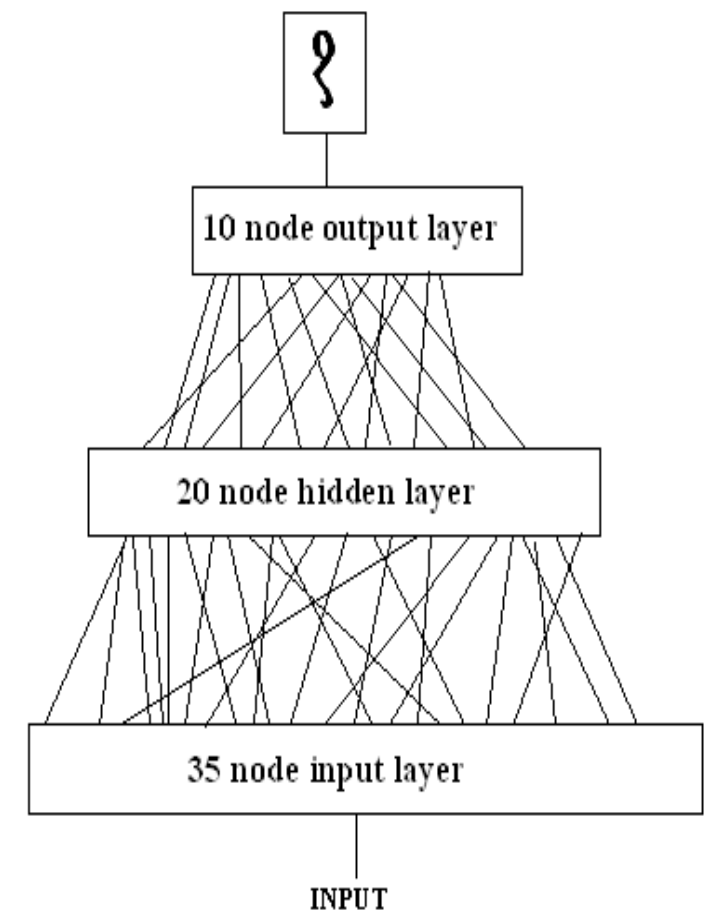

Fig. 9: Three layer artificial neural network for numeral recognition

The neural network algorithm trains the 50 numerals and the performance goal is met for 196 epochs as shown in Fig.10.

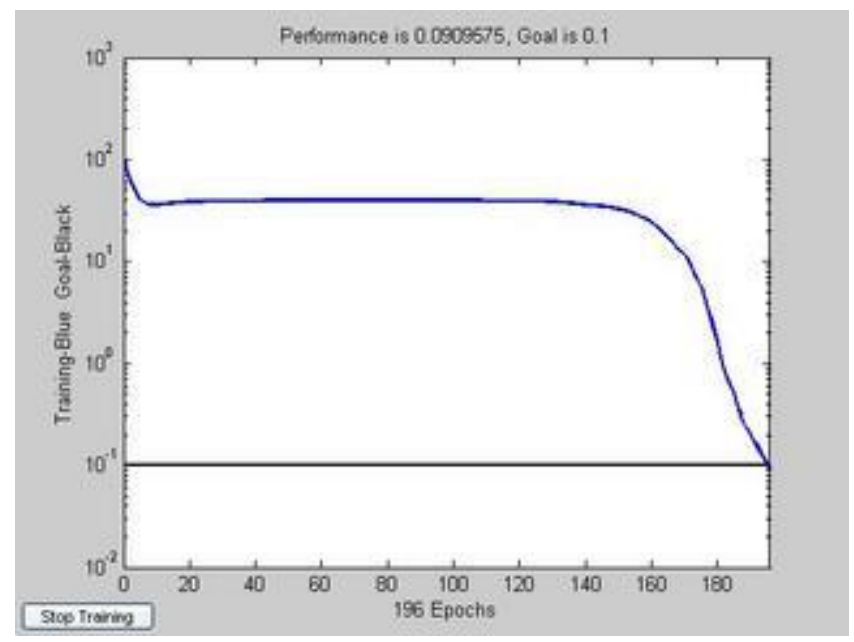

Fig. 10: Performance goal is met for 196 epochs

The test data of 100 numerals were used for testing purpose and the display gives the Marathi numerals as shown in Fig.11

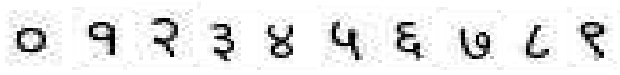

Fig. 11: Displayed result 


\section{Experimental Result}

Table 1 shows the experimental result for 100 Marathi numerals. In this table the applied Marathi numerals are 100 in numbers i.e. 10 samples of each number from ( 0 to 9$)$. The recognition accuracy achieved is approximately $97 \%$ for vector size $(5 \times 7)$ and $100 \%$ for vector size $(10 \times 14)$.

Table 1: Experimental result and percentage recognition

\begin{tabular}{|c|c|c|c|}
\hline Applied Marathi Numerals & $\begin{array}{l}\text { Numerals recognized } \\
\text { properly }\end{array}$ & $\begin{array}{l}\text { Percentage recognition for the } \\
\text { resolution of the input Vector } \\
\qquad(5 \times 7) \text {. }\end{array}$ & $\begin{array}{l}\text { Percentage recognition } \\
\text { for the resolution of } t \text { he } \\
\text { input Vector (10X14). }\end{array}$ \\
\hline 0 & 10 & $100 \%$ & $100 \%$ \\
\hline 9 & 10 & $100 \%$ & $100 \%$ \\
\hline २ & 10 & $100 \%$ & $100 \%$ \\
\hline ३ & 9 & $90 \%$ & $100 \%$ \\
\hline 8 & 10 & $100 \%$ & $100 \%$ \\
\hline 4 & 10 & $100 \%$ & $100 \%$ \\
\hline$\xi$ & 9 & $90 \%$ & $100 \%$ \\
\hline ט & 10 & $100 \%$ & $100 \%$ \\
\hline$\iota$ & 10 & $100 \%$ & $100 \%$ \\
\hline$\rho$ & 9 & $90 \%$ & $100 \%$ \\
\hline
\end{tabular}

\section{Conclusions}

It can be concluded that the Marathi 150 numerals can be trained and recognized properly. The $97 \%$ recognition result can be achieved for the resolution of the input vector $(5 \times 7)$. If some noise were introduced because of different writing styles of different persons from school children to a Doctorate person or by pen tips, the network can be treated for a longer time or retained with more neurons in its hidden layer. Also, the resolution of the input vector can be increased to a $(10 \mathrm{x}$ 14). The $100 \%$ recognition result has been achieved for the resolution of the input vector (10x 14).

\section{References}

[1] Patil S.B. and Sinha G.R, 'Off-line mixed Devnagri numeral recognition using Artificial Neural Network, Advance in computational Research, Bio info journal,ISSN:0975-3273 \& EISSN: 0975-9085, Volume 4, Issue1, 16-17 March 2012,pp. 38-41.

[2] Sandeep B. Patil, Dr. G.R. Sinha and Vaishali S. Patil, "Isolated Handwritten Devnagri Numerals Recogntion Using HMM", IEEE Catalog Number: CFP1172M-PRT. EAIT in Second International conference on Emerging Applications of
Information technology, Kolkota, ISBN: 978-14244-9683-9, pp.185-189.

[3] Sandeep B. Patil, G.R. Sinha and Kavita Thakur, 'Isolated Handwritten Devnagri Character Recognition Using Fourier Descriptor and HMM' in International Journal of Pure and Applied Sciences and Technology (IJPAST), ISSN:22296107, Volume 8, No 1, January 2012, pp. 69-74.

[4] Alain Biem, "Minimum classification Error Training for on line Handwriting Recognition", IEEE Transaction on Pattern analys is and machine intelligence, 28(7): 1041-1051, 2006.

[5] Deniz Erdogmus, Oscar Fontenlu-Romeo, Jose C. Principe, " Linear-least-squares Initialization of Multilayer Perceptrons Through Backpropogation of the desired response", IEEE Tranaction on Neural network, 16(2): 325-337, 2005.

[6] Rafael C. Gonzalez and Richard E. Woods, "Dig ital Image Processing Using Matlab", Pearson Education, India, 2006.

[7] Jarek M. Zarada, "Introduction to artificial neural system”, Jaico publishing house, 1999.

[8] Anil K.Jain, "Fundamentals of Digital Image processing”, PHI publication, New Delhi, 1995. 
[9] L. Fausett, "Fundamentals of Neural Networks Architectures, Algorithms, and Applications", Prentice Hall Publication, 2002.

[10] T. Fontaine and L. Shastri, "Handprinted digit recognition using spatiotemporal connectionist models," In Proceedings of the IEEE Conference on Computer Vision and Pattern Recognition, 1999.

[11] S. K. Parui and B. Shaw., "Offline handwritten devanagari word recognition: An $\mathrm{hmm}$ based approach", In Proc. Of PReMI-2007(Springer), LNCS-4815:528-535, December 2007.

[12] L. R. Rabiner, " A tutorial on hidden markov models and selected application in speech recognition", In Proceedings of the IEEE. 77(2):257-286, February 1989.

[13] Magdi A. Mohamed and Paul Gader, "'Generalized Hidden Markov Models-Part II: Application to Handwritten Word Recognition", IEEE Trans. On Fuzzy System, 8(1): 123-129, February 2000.

[14] Jia Zeng and Zhi-Qiang Liu, “Markov Random Field-Based Statistical Character Structure Modeling for Handwritten Chinese Character Recognition", IEEE Trans. On Pattern analysis and Machine aintelligence, 30(5): 345-349, 2008.

[15] Stephan R. Veltman and Ramjee Prasad, "Hidden Morkov Models Applied to On-Line Handwritten Isolated Character Recognition", IEEE Trans. On Image Processing, 3(3): 1994.

[16] H. Bentounsi, M. Batouche, "Incremental support vector machines for handwritten Arabic character recognition". Proceeding of the international conference on information and communication Technologies, 2004, pp 1764-1767.

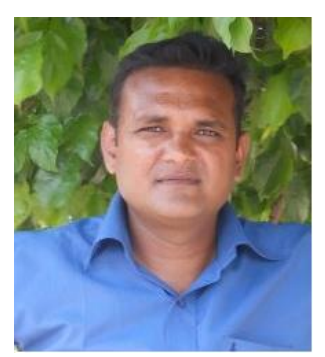

Mr. S andeep B. Patil, received his B.E. (E\&Tc) and M.E.(Elex) from Dr. B.A.M.U. Aurangabad (M.S). He is a research scolar and pursuing his Ph.D. in the field of image processing from CSVTU Bhilai. He is working as Associate Professor in the department of Electronics \& Telecommunication of Shri Shankaracharya Group of institutions Bhilai. He has published 15 research papers in various National and International journals and conferences. His research interest include Biometrics and its applications, Image Processing and applications such as Devnagri character recognition Using neural Network, PCA, HMM, and Pattern recognition.

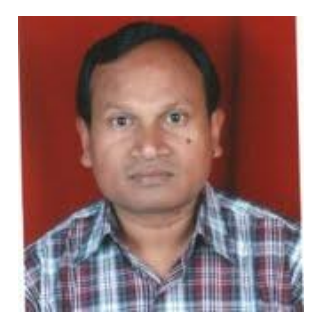

Dr. G.R.Sinha, B.E., M.Tech. (Gold Medalist), Ph.D. (Electronics Telecom.), MIEEE, AMIETE, AMIE, LMISTE, MCSI. He is working as Associate Director in SSGI and Professor in (Electronics\& Telecomm. Deptt.) of SSGI Bhilai.. He is also recipient of Chhattisgarh Young scientist award, Expert Engineer Award, Nominated for ISCA Young scientist award. He has published 94 research papers in various international and national journals and conferences. His areas of interest includes image processing, biomedical imaging, nano-imaging, neural network, fuzzy logic, Pattern recognition.

How to cite this paper: Sandeep B. Patil, G.R. Sinha,"Real Time Handwritten Marathi Numerals Recognition Using Neural Network", International Journal of Information Technology and Computer Science(IJITCS), vol.4, no.12, pp.76-81, 2012. DOI: $10.5815 /$ ijitcs.2012.12.08 